\title{
Probabilistic distance-based quantizer design for distributed estimation
}

\author{
Yoon Hak Kim iD
}

\begin{abstract}
We consider an iterative design of independently operating local quantizers at nodes that should cooperate without interaction to achieve application objectives for distributed estimation systems. We suggest as a new cost function a probabilistic distance between the posterior distribution and its quantized one expressed as the Kullback Leibler (KL) divergence. We first present the analysis that minimizing the $\mathrm{KL}$ divergence in the cyclic generalized Lloyd design framework is equivalent to maximizing the logarithmic quantized posterior distribution on the average which can be further computationally reduced in our iterative design. We propose an iterative design algorithm that seeks to maximize the simplified version of the posterior quantized distribution and discuss that our algorithm converges to a global optimum due to the convexity of the cost function and generates the most informative quantized measurements. We also provide an independent encoding technique that enables minimization of the cost function and can be efficiently simplified for a practical use of power-constrained nodes. We finally demonstrate through extensive experiments an obvious advantage of improved estimation performance as compared with the typical designs and the novel design techniques previously published.

Keywords: Distributed compression, Distributed source coding (DSC), Quantizer design, Posterior distribution, KL divergence, Generalized Lloyd algorithm, Source localization, Sensor networks

Abbreviations: ARE, Asymptotic relative efficiency; DOQ, Distributed optimized quantizer; DSC, Distributed source coding; EDQ, Equally distance-divided quantizer; KL, Kullback Leibler; LE, Localization error; LSQ, Localization-specific quantizer; ML, Maximum likelihood; PDQ, Probabilistic distance-based quantizer; PDQ-R, Probabilistic distance-based quantizer-reduced; R-D, Rate-distortion; SFQ, Score-functional quantizer; SNR, Signal-to-noise ratio
\end{abstract}

\section{Introduction}

In distributed estimation systems where spatially separated sensor nodes are battery-powered and operate under strict limitations on wireless communication bandwidth, the sensor nodes measure the parameter of interest, quantize their measurements, and send the quantized data to a fusion node which then performs estimation of the parameter. It is reported that the rate-distortion performance can be greatly improved by adopting efficient quantizers at nodes as compared with simple uniform quantizers.

In distributed source coding (DSC) framework where nodes at different locations collect data and transmit them

Correspondence: yhk@chosun.ac.kr

Department of Electronic Engineering, College of Electronic \& information Engineering, Chosun University, 309 Pilmun-daero, Dong-gu, 61452 Gwangju, Korea to a fusion node, practical design techniques for quantizers have been reported [1-3]: to achieve the Wyner-Ziv bound, trellis codes for DSC that are computationally efficient were proposed in [1]. An iterative design for quantizer in the Lloyd algorithm framework was shown to further reduce the distortion by producing the nonregular scalar quantizers, implying that several disjoint intervals can be mapped to a single codeword [2]. In addition, since various practical design algorithms have been developed in the Lloyd algorithm framework, they can be typically affected by initialization of quantizers, leading to numerous poor local optima. Thus, to overcome this, an iterative algorithm was proposed by using a deterministic annealing technique for a robust DSC system [3]. An iterative algorithm for construction of the optimal quantization partitions was studied in distributed estimation systems [4] where computation of the estimator function used in encoding of such partitions may be 
practically prohibitive. To avoid the encoding complexity, a suboptimal approach (i.e., linear estimator) was considered for quantizer design [5]. Under the assumption of one-bit message from nodes to fusion node, universal decentralized estimation schemes were investigated [6] and distributed estimators were derived for pragmatic signal models [7]. A brief investigation for quantization in distributed estimation and classification systems was presented in [8].

Since standard quantization focuses on minimization of a local metric (e.g., reconstruction error of local sensor readings), it should be modified to optimize at each step a global metric such as estimation error: more specifically, the two main tasks in the Lloyd design framework (i.e., quantization partition construction and the corresponding codeword computation) should be dedicated to minimizing the estimation error. However, the design difficulty arises since the quantization partitions constructed to minimize the estimation error are not generally independently encodable at each node: that is, encoding (or mapping) of local measurements into one of the quantization partitions would be possible after computing the global metric, which is not accessible at each node where only local measurements are available.

To circumvent the difficulty, a distributional distance between the distributions under two hypotheses was suggested as a global metric for quantizer design to yield a manageable design procedure [9]. A distance-based metric to measure the loss due to quantization was adopted for uniform or nonuniform quantizers in the high-resolution regime [10]. A vector quantization technique that minimizes the Kullback Leibler (KL) divergence was proposed for distribution matching [11]. An iterative design algorithm that maximizes the minimum asymptotic relative efficiency (ARE) was proposed, illustrating that the score-functional quantizer (SFQ) would be optimal so as to maximize the minimum ARE for distributions with the monotonicity property [12]. For acoustic sensor networks, a distance error at nodes was proposed in the functional quantization framework to ensure convergence in an iterative process [13]. A weighted sum of both of the metrics was proposed as a cost function (i.e., local $+\lambda \times$ global) along with a search for proper weights that guarantee construction of the encodable quantization partitions while maintaining the non-increasing cost function at iterations [14], showing a significant performance gain over typical designs. To reduce design complexity, efficient algorithms that allow us to search sequentially the boundary values of the quantization intervals in scalar quantizers have been developed $[15,16]$.

It was also observed in [17] that multiple disjoint quantization bins at nodes can be merged to a single bin or a single codeword without performance loss for distributed estimation systems. The merging technique determines the true bin transmitted from the node of interest by taking into account the measurements from other nodes, hence achieving a significant rate reduction. In addition, an iterative quantizer design algorithm was proposed [18] to incorporate non-regularity into design process, implying the correspondence between multiple disjoint partitions and a single codeword: specifically, the bins (e.g., intervals in scalar quantizers) are regarded as the elements that will be processed for mapping to their corresponding codewords (or reconstruction values), resulting in disjoint Voronoi regions (e.g., union of multiple intervals in scalar quantizers). Recently, a novel encoding scheme of assigning multiple codewords to each quantization partition was proposed to implement a low-weight independent encoding of the optimal partitions [19].

In this paper, we consider an iterative design of independently operating local quantizers at nodes in the Lloyd algorithm framework. Instead of directly minimizing the estimation error, we choose to use an indirect metric related to the posterior distribution. Specifically, we define quantization of the posterior distribution and focus on minimizing the probabilistic distance between the posterior distribution and its quantized version as a global cost function. We first express the cost function as the KL divergence which typically causes a high computational cost. We develop a feasible design procedure by presenting the analysis that minimizing the KL divergence is equivalently reduced to maximizing the logarithmic quantized posterior distribution on the average which is properly further simplified as a new cost function, yielding a substantial reduction in design complexity. We discuss that the proposed algorithm converges to a global optimum due to the convexity of the cost function in the quantized posterior distribution [20], which is experimentally examined by showing that our design operates robust under various test conditions. We also show that the proposed quantizer generates the most informative quantized measurements, which would be efficiently used by estimation techniques at fusion node to improve the estimation performance.

We highlight that the independent encoding minimizing the global cost function can be accomplished and also provide an efficient approximation to the encoding technique to avoid a computational burden at each node in practical use while maintaining a reasonable estimation performance. Note that most of the previous work conduct encoding of local measurements by simply computing the local Euclidean distance between sensor readings and codewords. We finally demonstrate through extensive simulations that the proposed algorithm performs well with respect to the previously developed techniques $[14,18]$ owing to the two main advantages of our proposed algorithm such as the global optimality and the encoding technique designed to optimize the system-wide 
metric. In this work, it is assumed that the sensor nodes do not exchange data with each other; they send their measurements to a fusion node via reliable communication links.

This paper is organized as follows. The problem formulation of the quantizer design is given in Section 2. A new cost function is introduced and properly incorporated into our iterative design algorithm in Section 3. Discussions of performance of the proposed algorithm and design complexity for our encoding technique are provided in Section 3.1 and Section 3.2. An application example for the proposed algorithm is briefly presented in Section 4. Simulation results are given in Section 5, and the conclusions are found in Section 6.

\section{Problem formulation}

We consider a distributed estimation system where $M$ sensor nodes are randomly deployed at known spatial locations, $\mathbf{x}_{i} \in \mathbf{R}^{2}, i=1, \ldots, M$. Each node senses the signals generated from the unknown parameter $\theta \subset \mathbf{R}^{N}$ and sends its measurement to a fusion node for estimation of the parameter. Assuming the sensing model $f_{i}\left(\theta, \mathbf{x}_{i}\right)$ employed at node $i$, the measurement at node $i$ denoted by $z_{i}$ can be expressed as follows:

$$
z_{i}(\theta)=f_{i}\left(\theta, \mathbf{x}_{i}\right)+\omega_{i}, \quad i=1, \ldots, M
$$

where the measurement noise $\omega_{i}$ is assumed to be approximated by normal distribution $N\left(0, \sigma_{i}^{2}\right)$, and the measurements are also assumed to be statistically independent of each other given the parameter; that is, $p\left(z_{1}, \cdots z_{M} \mid \theta\right)=$ $\prod_{i=1}^{M} p\left(z_{i} \mid \theta\right)$. Each node uses an $R_{i}$-bit quantizer with quantization level $L_{i}=2^{R_{i}}$ and the dynamic range $D_{i}=\left[\begin{array}{ll}z_{i}^{\min } & z_{i}^{\max }\end{array}\right]$. Note that the quantization range $D_{i}$ can be determined for nodes, based on their respective sensing ranges. Each node quantizes its measurement and generates the codeword $\hat{z}_{i}$ for $z_{i}$ according to its encoding rule (e.g., minimum Euclidean distance rule). For example, if the measurement $z_{i}$ belongs to the $j$ th quantization partition $V_{i}^{j}$ by using its encoding rule, the node $i$ will transmit the $j$ th codeword $\hat{z}_{i}^{j}$ to a fusion node which produces an estimate of the parameter, $\hat{\theta}$ from the received quantized measurements, $\hat{z}_{i}, i=1, \ldots, M$ from all nodes.

Notation: A large proportion of our notation will be introduced as needed. However, a couple of basic notations will be given now: the bold characters $\mathbf{z}_{1}^{M}$ and $\hat{\mathbf{z}}_{1}^{M}$ indicate a vector of measurements $\left(z_{1}, \cdots, z_{M}\right)$ and a vector of codewords $\left(\hat{z}_{1}, \cdots, \hat{z}_{M}\right)$, respectively, and the parameter $\theta$ is treated as a vector of parameters $\left(\theta_{1}, \cdots, \theta_{N}\right)$. In addition, $\mathbf{z}_{1 / i}^{M}$ is the shortened notation for a vector of $M \quad-1$ measurements $\left(z_{1}, \cdots, z_{i-1}, z_{i+1}, \cdots, z_{M}\right)$, implying that the subscript $i$ indicates the element omitted from the set of measurements.

\subsection{Criteria for quantizer optimization}

Obviously, quantizers optimized in the Lloyd framework for distributed estimation systems should seek to minimize the estimation error, $\|\theta-\hat{\theta}\|^{2}$ which a function of all of the codewords generated from $M$ nodes involved. Thus, the quantization partitions and their corresponding codewords are iteratively generated to reduce the estimation error at each step while such quantization partitions remain independently encodable at each node.

To ensure the independent encoding and minimization of the estimation error which are the two crucial conditions for quantizer design algorithms in distributed estimation systems, several global metrics related to the estimation error were previously developed: the distributional distance [9] for distributed detection and the global distance function for distributed estimation [13]. In this perspective, we suggest quantization of the posterior distribution $p\left(\theta \mid \mathbf{z}_{1}^{M}\right)$ and seek to design local quantizers that minimize the probabilistic distance between $p\left(\theta \mid \mathbf{z}_{1}^{M}\right)$ and its quantized distribution which can be expressed as the KL divergence [20]. We show that using the distance as a new cost function provides several benefits for quantizer design in distributed estimation: first, minimizing the probabilistic distance results in quantizers that generate the codewords maximizing the logarithmic quantized posterior distribution $\log p\left(\theta \mid \hat{\mathbf{z}}_{1}^{M}\right)$ on the average, thus improving the estimation accuracy. Second, the independent encoding can be efficiently performed since the probabilistic distance is computed based on $p\left(\theta \mid \mathbf{z}_{1}^{M}\right)$ and $M$ quantizers, not requiring the actual measurements at the other nodes. Third, it could allow us to establish a global encoding of local measurements into their quantization partitions which would not be achieved by typical encoding rules (e.g., minimum Euclidian distance rule) used for previous novel design techniques. The benefits of our algorithm will be elaborated in the following sections.

\section{Quantizer design algorithm}

We consider for a given rate $R_{i}, i=1, \cdots, M$ the problem of designing independent local quantizers that minimize the KL divergence between the posterior distribution $p\left(\theta \mid \mathbf{z}_{1}^{M}\right)$ and its quantized one denoted by $q\left(\theta \mid \mathbf{z}_{1}^{M}\right)$ which is defined from quantization of $p\left(\theta \mid \mathbf{z}_{1}^{M}\right)$ : formally,

$$
q\left(\theta \mid \mathbf{z}_{1}^{M}\right)=p\left(\theta \mid \hat{\mathbf{z}}_{1}^{M}\right), \quad Q_{i}\left(z_{i}\right)=\hat{z}_{i}, i=1, \cdots, M
$$

where $Q_{i}$ indicates the quantizer employed at node $i$. 
First, we simplify our metric denoted by $D_{\mathrm{KL}}\left[p\left(\theta \mid \mathbf{z}_{1}^{M}\right) \| q\left(\theta \mid \mathbf{z}_{1}^{M}\right)\right]$ to avoid unnecessary computations for quantizer design at each node. By definition of the $\mathrm{KL}$ divergence, we have

$$
\begin{aligned}
D_{\mathrm{KL}} & =\sum_{\theta} p(\theta) \sum_{\mathbf{z}_{1}^{M}} p\left(\mathbf{z}_{1}^{M} \mid \theta\right) \frac{\log p\left(\theta \mid \mathbf{z}_{1}^{M}\right)}{\log q\left(\theta \mid \mathbf{z}_{1}^{M}\right)} \\
& =E_{\theta, \mathbf{z}_{1}^{M}} \log p\left(\theta \mid \mathbf{z}_{1}^{M}\right)-E_{\theta, \mathbf{z}_{1}^{M}} \log q\left(\theta \mid \mathbf{z}_{1}^{M}\right)
\end{aligned}
$$

Noting that the first term is irrelevant to minimization of the metric over quantizers $Q_{i}$, we can find the quantizers $\mathbf{Q}^{*}=\left[Q_{1}, \cdots, Q_{M}\right]$ minimizing the $\mathrm{KL}$ divergence as follows:

$$
\begin{aligned}
\mathbf{Q}^{*} & =\arg \max _{Q_{1}, \cdots, Q_{M}} E_{\theta, \mathbf{z}_{1}^{M}} \log q\left(\theta \mid \mathbf{z}_{1}^{M}\right) \\
& =\arg \max _{Q_{1}, \cdots, Q_{M}} E_{\mathbf{z}_{1}^{M}} E_{\theta \mid \mathbf{z}_{1}^{M}} \log q\left(\theta \mid \mathbf{z}_{1}^{M}\right)
\end{aligned}
$$

Thus, our problem is reduced to that of designing a set of quantizers that maximize the metric $E_{\theta, \mathbf{z}_{1}^{M}} \log q\left(\theta \mid \mathbf{z}_{1}^{M}\right)$.

It should be noticed that we optimize a quantizer at each node, while quantizers for the other nodes remain unchanged. This is done successively for each sensor node and repeated over all nodes until a stopping criterion is satisfied. This notion allows us to make a further simplification of the metric for faster computation by removing irrelevant terms:

$$
\begin{aligned}
& E_{\theta, \mathbf{z}_{1}^{M}} \log q\left(\theta \mid \mathbf{z}_{1}^{M}\right) \\
& =E_{\theta, \mathbf{z}_{1}^{M}}\left[\log q\left(\mathbf{z}_{1}^{M} \mid \theta\right)+\log p(\theta)-\log q\left(\mathbf{z}_{1}^{M}\right)\right] \\
& =E_{\theta, \mathbf{z}_{1}^{M}}\left[\log q\left(\mathbf{z}_{1 / i}^{M} \mid \theta\right)+\log p(\theta)+\log q\left(z_{i} \mid \theta\right)-\log q\left(\mathbf{z}_{1}^{M}\right)\right] \\
& \propto E_{\theta, \mathbf{z}_{1}^{M}}\left[\log q\left(z_{i} \mid \theta\right)-\log q\left(\mathbf{z}_{1}^{M}\right)\right]
\end{aligned}
$$

where (5) follows from independence of $\mathbf{z}_{1}^{M}$ given the parameter and (6) follows from the observation that the first and the second terms in (5) are irrelevant for quantizer design at node $i$. Note that $q=p\left(\hat{z}_{i}^{j} \mid \theta\right)$ when $z_{i}$ is assigned to the $j$ th quantization partition or the $j$ th codeword.

Now, we are in a position to consider the quantizer design process in the generalized Lloyd design framework. First, we construct the Voronoi region so as to maximize (6) as follows:

$$
\begin{aligned}
V_{i}^{j} & =\left\{z_{i}: E_{\theta}\left[p\left(z_{i} \mid \theta\right)\left(\log p\left(\hat{z}_{i}^{j} \mid \theta\right)-E_{\mathbf{z}_{1 / i}^{M} \mid \theta} \log p\left(\hat{\mathbf{z}}_{1 / i}^{M}, z_{i}=\hat{z}_{i}^{j}\right)\right)\right]\right. \\
& \left.\geq E_{\theta}\left[p\left(z_{i} \mid \theta\right)\left(\log p\left(\hat{z}_{i}^{k} \mid \theta\right)-E_{\mathbf{z}_{1 / i}^{M} \mid \theta} \log p\left(\hat{\mathbf{z}}_{1 / i}^{M}, z_{i}=\hat{z}_{i}^{k}\right)\right)\right], \forall k \neq j\right\}
\end{aligned}
$$

where $p\left(\hat{z}_{i}^{j} \mid \theta\right)$ is given by $p\left(z_{i}=\hat{z}_{i}^{j} \mid \theta\right) \sim N\left(f_{i}(\theta), \sigma_{i}^{2}\right)$.
Second, we compute the codeword corresponding to $V_{i}^{j}$ in a similar manner:

$$
\begin{aligned}
\hat{z}_{i}^{*}= & \arg \max _{\hat{z}_{i} \in D_{i}} E_{\theta}\left[\sum _ { z _ { i } \in V _ { i } ^ { j } } p ( z _ { i } | \theta ) \left(\log p\left(\hat{z}_{i} \mid \theta\right)\right.\right. \\
& \left.-E_{\mathbf{z}_{1 / i}^{M} \mid \theta} \log p\left(\hat{\mathbf{z}}_{1 / i}^{M}, z_{i}=\hat{z}_{i}\right)\right]
\end{aligned}
$$

It should be observed that in real situations, local quantizers should operate independent of other quantizers. Hence, an independent encoding would be a crucial requirement for such quantizer designs. We present the encoding technique that assigns a local measurement $z_{i}$ to one of the quantizer partitions so as to maximize the metric employed in the design as follows:

$$
\begin{aligned}
V_{i}^{j *}= & \arg \max _{1 \leq j \leq L_{i}} E_{\theta}\left[p ( z _ { i } | \theta ) \left(\log p\left(\hat{z}_{i}^{j} \mid \theta\right)\right.\right. \\
& \left.\left.-E_{\mathbf{z}_{1 / i}^{M} \mid \theta} \log p\left(\hat{\mathbf{z}}_{1 / i}^{M}, z_{i}=\hat{z}_{i}^{j}\right)\right)\right]
\end{aligned}
$$

Obviously, the encoding process in (9) is carried out by using $p\left(\mathbf{z}_{1}^{M} \mid \theta\right)$ and $M$ quantizers without requiring actual measurements $\mathbf{z}_{1 / i}^{M}$ at the other nodes.

\subsection{Remarks on optimality and performance}

Since the proposed algorithm is conducted in the generalized Lloyd design framework, it would suffer from numerous poor local optima. However, the metric (3) is shown to be convex in the quantized distribution $q\left(\theta \mid \mathbf{z}_{1}^{M}\right)$ given $p\left(\theta \mid \mathbf{z}_{1}^{M}\right)$ (see [20] for the proof), implying that any local minimum must be a global minimum. In addition, the quantized distribution is uniquely determined by quantizers (refer to (2)) and designing quantizers that reduce the metric at each step is equivalent to finding the corresponding quantized distributions. Thus, it is concluded that our algorithm always results in quantizers that globally minimize the metric and thus provide robustness to various design factors.

It has been also shown from (4) that minimizing $D_{\mathrm{KL}}$ over quantizers is equivalent to maximizing the average logarithmic quantized posterior distribution. For example, suppose that given two different sets of $M$ quantizers, say $\mathbf{Q}_{1}^{M}$ and $\tilde{\mathbf{Q}}_{1}^{M}$ where $\mathbf{Q}_{1}^{M}$ indicates our proposed quantizers given by (4), a certain parameter $\theta$ is sensed by $M$ nodes, which in turn generate $\hat{\mathbf{z}}_{1}^{M}$ and $\hat{\tilde{\mathbf{z}}}_{1}^{M}$, respectively. Then, it can be stated that the proposed quantizers generate better quantized measurements in a sense that $\log p\left(\theta \mid \hat{\mathbf{z}}_{1}^{M}\right) \geq \log p\left(\theta \mid \hat{\mathbf{z}}_{1}^{M}\right)$ on the average. In a different perspective, the performance of the proposed quantizers would be further examined by rewriting our metric for 
each $\mathbf{z}_{1}^{M}$ and simplifying it in the high-resolution regime as follows:

$$
\begin{aligned}
\mathbf{Q}^{*} & =\arg \max _{Q_{1}, \cdots, Q_{M}} \sum_{\theta} p\left(\theta \mid \mathbf{z}_{1}^{M}\right) \log q\left(\theta \mid \mathbf{z}_{1}^{M}\right) \\
& =\arg \max _{Q_{1}, \cdots, Q_{M}} \sum_{\theta} p\left(\theta \mid \mathbf{z}_{1}^{M}\right) \log p\left(\theta \mid \hat{\mathbf{z}}_{1}^{M}\right) \\
& \approx \arg \max _{Q_{1}, \cdots, Q_{M}} \sum_{\theta} p\left(\theta \mid \hat{\mathbf{z}}_{1}^{M}\right) \log p\left(\theta \mid \hat{\mathbf{z}}_{1}^{M}\right) \\
& =\arg \min _{Q_{1}, \cdots, Q_{M}} H\left(\theta \mid \hat{\mathbf{z}}_{1}^{M}=\mathbf{Q}_{1}^{M}\left(\mathbf{z}_{1}^{M}\right)\right)
\end{aligned}
$$

where (10) follows from the definition of $q\left(\theta \mid \mathbf{z}_{1}^{M}\right)$, (11) is derived from the high-resolution assumption, and (12) is obtained from the definition of the conditional entropy $H\left(\theta \mid \hat{\mathbf{z}}_{1}^{M}\right)$. Since the entropy can be minimized by choosing the most informative distributions $p\left(\theta \mid \hat{\mathbf{z}}_{1}^{M}\right)$, our quantizers would generate the most informative quantized measurements, yielding a good estimation accuracy which will be investigated by conducting extensive experiments in Section 5.

\subsection{Reduction of encoding complexity}

It should be emphasized that one of the benefits of our algorithm is the encoding technique that operates on local measurements and at the same time optimizes our global metric whereas most of the previous designs employ the minimum Euclidean distance rule to independently assign local measurements to the predetermined quantization partitions. In our design, encoding of $z_{i}$ to one of the partitions is independently executed in a system-wide sense that the metric $E \log q\left(\theta \mid \mathbf{z}_{1}^{M}\right)$ is maximized, although such encoding requires a high computational cost at nodes.

In this section, we consider a computational reduction in the encoding complexity for a practical use of powerconstrained sensor nodes in distributed systems. Noting that given $z_{i}$, the region of $\theta$ denoted by $A_{\theta}\left(z_{i}\right)$ with $p(\theta \in$ $\left.A_{\theta}\left(z_{i}\right) \mid z_{i}\right) \approx 1$ can be easily constructed, the independent encoding in (9) could be approximately conducted as follows:

$$
\begin{aligned}
V_{i}^{j *} \approx & \arg \max _{j} E_{\theta \in A_{\theta}}\left[p ( z _ { i } | \theta ) \left(\log p\left(\hat{z}_{i}^{j} \mid \theta\right)\right.\right. \\
& \left.\left.-E_{\mathbf{z}_{1 / i}^{M} \in B_{\theta} \mid \theta} \log p\left(\hat{\mathbf{z}}_{1 / i}^{M}, z_{i}=\hat{z}_{i}^{j}\right)\right)\right]
\end{aligned}
$$

where $B_{\theta}$ is a set of the measurements at other nodes and can be substantially reduced again by using $A_{\theta}$ : that is, $B_{\theta} \approx\left\{z_{1 / i}^{M}(\theta): \theta \in A_{\theta}\right\}$. This further approximation will reduce the encoding complexity dramatically.

\subsection{Summary of algorithm}

The design algorithm at node $i$ is summarized as follows and is iteratively executed over all sensor nodes $i=1, \ldots, M$.
Algorithm 1 Iterative quantizer design algorithm at sensor $i$

Step1: Initialize the quantizer $Q_{i}\left(z_{i}\right)$. Set thresholds $\epsilon$ and iteration index $\kappa=1$.

Step2: Construct the quantization partition $V_{i}^{j}, j=$ $1, \cdots, L_{i}$ using (7).

Step3: Compute the codeword for each partition using (8).

Step4: Compute the metric in (6) denoted by $P_{\kappa}$.

Step5: If $\frac{P_{\kappa}-P_{\kappa}-1}{P_{\kappa}}<\epsilon$ stop; otherwise continue.

Step6 : Replace $\hat{z}_{i}^{j}$ by $\hat{z}_{i}^{*}$, Set $\kappa=\kappa+1$ and go to Step 2 .

\section{Application of quantizer design algorithm}

In this section, as an application system of our design algorithm, we briefly introduce a source localization system where $M$ nodes equipped with acoustic amplitude sensors measure signal energy generated from a source located at an unknown location $\theta \in \mathbf{R}^{2}$ and quantize the measurements before sending them to a fusion node for localization. In expressing the signal energy measured at nodes, we adopt an energy decay model which was proposed and experimentally verified in [21] and employed in $[22,23]$. The signal energy measured at node $i$ denoted by $z_{i}$ can be expressed as follows:

$$
z_{i}(\theta)=g_{i} \frac{a}{\left\|\theta-\mathbf{x}_{i}\right\|^{\alpha}}+w_{i}
$$

where $g_{i}$ is the gain factor at node $i$ and $\alpha$ is the energy decay factor which is approximately equal to 2 in free space. Note that a sound source generates acoustic energy which will attenuate at a rate inversely proportional to the square of the distance in free space [24]. The signal energy $a$ which can be jointly estimated with the source location [25] is assumed to be known during localization process. It is also assumed that the measurement noise $w_{i}$ can be approximated using a normal distribution, $N\left(0, \sigma_{i}^{2}\right)$.

\section{Simulation results}

In this section, we design the proposed quantizers using training sets in which source locations are assumed to be uniformly distributed and the local measurements are collected from the model parameters $\alpha=2, g_{i}=1$, and $a=50$ in a noiseless condition $\sigma_{i}^{2}=\sigma^{2}=0$. In testing our quantizers, we apply two encoding techniques in (9) and (13), denoted by probabilistic distance-based quantizer (PDQ) and PDQ-reduced (PDQ-R), respectively. In the experiments, we first consider a sensor network where $M(=5)$ sensors are deployed in a $10 \times 10 \mathrm{~m}^{2}$ sensor field. For each of 100 different sensor configurations, we design uniform quantizers (Unif Q), Lloyd quantizers (Lloyd Q), and several novel quantizers for $R_{i}=2,3,4$ and evaluate them by generating a test set of 1000 source locations from the model parameters which were assumed during quantizer design. 
Experiments are extensively conducted to investigate the effectiveness of different design algorithms and the sensitivity to parameter perturbation and variation of noise level. Furthermore, since typical sensor networks employ many sensor nodes in a large sensor field, we also consider a larger sensor field $20 \times 20 \mathrm{~m}^{2}$ to test our algorithm over typical designs. In the experiments, performance evaluation is carried out by comparing the average localization error $E\|\theta-\hat{\theta}\|^{2}$ computed from the maximum likelihood (ML) estimation technique for fast computation.

\subsection{Comparison with traditional quantizers}

First, our quantizer is compared with typical standard designs such as uniform quantizers and Lloyd Q in Fig. 1 where the localization error (meter) is averaged over 100 node configurations for each rate $R_{i}$. For a clear comparison, the overall rate-distortion (R-D) curves are depicted for the different quantizations. As expected, PDQ provides a significant performance gain over traditional quantizers since our proposed algorithm iteratively finds the probabilistic distance-based mapping that generates better quantized measurements in a sense of better quantized posterior distribution. It should be further noticed that PDQ-R also shows a considerable performance improvement, implying justification of the approximation to derive our low-complexity encoding technique in (13).

\subsection{Performance evaluation: comparison with the previous novel designs}

We further examine the performance of the proposed design algorithm by comparing with the previous novel

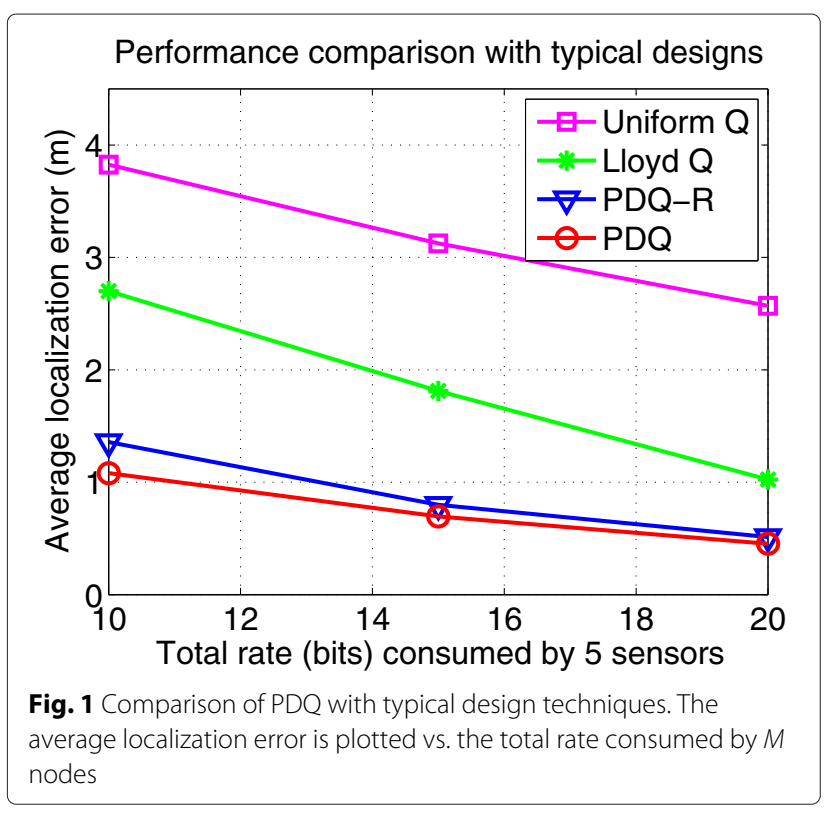

design techniques such as the localization-specific quantizer (LSQ) in [14] and the distributed optimized quantizer (DOQ) in [18]. Note that both of them have been developed as distributed source coding (DSC) techniques for distributed estimation systems and tested for source localization in acoustic amplitude sensor networks in the previous work. In designing quantizers, we initialize them with the equally distance-divided quantizer (EDQ) to avoid possibly poor local minima. Note that EDQ can be simply designed by uniformly dividing the sensing distance, not the dynamic range of the measurement. EDQ shows good localization performance so as to be used as an efficient initialization for quantizer design $[14,26]$.

In the experiments, we collect the two test sets from 1000 random source locations with the measurement noise $\sigma_{i}=0$ and $\sigma_{i}=0.15$, respectively, for evaluation. The R-D curves for the design techniques are illustrated in Fig. 2. Surprisingly, PDQ outperforms LSQ mainly because our algorithm enables the global encoding (in our case, probabilistic distance-based encoding) whereas LSQ operates by a regular encoding (i.e., minimum Euclidean distance rule). In addition, our quantizer performs well with respect to DOQ which adopts a non-regular mapping with a huge design complexity (see the details in [18]). Note that our algorithm focuses on minimization of the probabilistic distance caused by quantization, not directly optimizing the estimation accuracy. Nonetheless, PDQ offers a noteworthy performance improvement as compared with the previous novel designs, which can be explained from the analysis that our algorithm always produce a global optimum equipped with the powerful encoding technique whereas the others suffer from poor local optima that operate on a local distance rule.

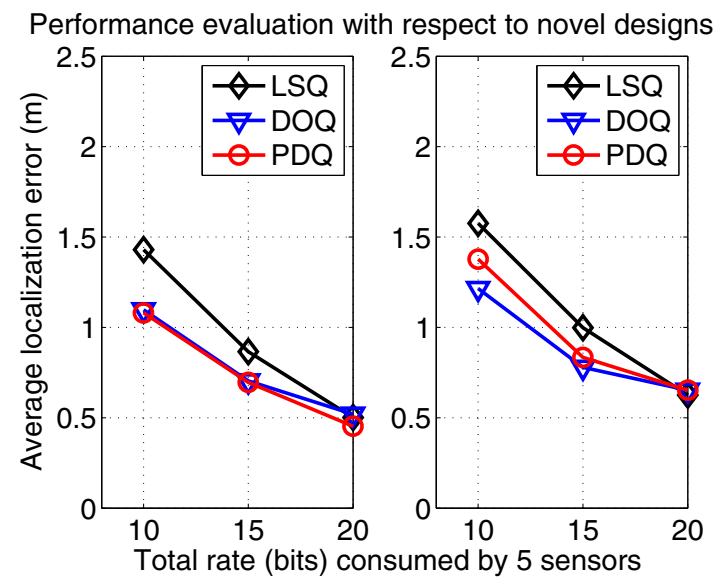

Fig. 2 Comparison of PDQ (PDQ-R) with novel design techniques. The average localization error in meter is plotted vs. the total rate (bits) consumed by five sensors with $\sigma=0$ (left) and $\sigma=0.15$ (right), respectively 


\subsection{Sensitivity analysis of design algorithms}

In this section, we first examine the proposed algorithm by making perturbation of the mode parameters from those assumed in the design stage. We further need to investigate the performance of the different design algorithms in the presence of the measurement noise since the quantizers are designed by using the training sets generated from the assumption of noiseless measurements $(\sigma=0)$. It could be expected that our proposed algorithm will show a strong robustness to various design factors since it pursues a global optimum.

\subsubsection{Sensitivity of PDQ to parameter perturbation}

In this experiment, PDQ is tested under various types of parameter perturbation. We varied one of the model parameters (i.e., decay factor $\alpha$ and gain factor $g_{i}$ ) from what was used during the training stage of quantizers for each test. It is assumed that the true parameters are available at a fusion node for localization to inspect only the effect of the quantizer design on the localization performance. Note that the assumption is quite reasonable since the localization algorithms provide good robustness to the parameter perturbation (see [25]). The experimental results are given in Table 1 . As expected, PDQ shows better robustness to variation of the gain factor than that of the decay factor since the latter causes more severe distortion in local measurements. Obviously, our design operates very reliably in the presence of a small perturbation of the model parameters.

\subsubsection{Sensitivity of design algorithms to noise level}

In this experiment, we study the sensitivity of various design algorithms to noise level. For each configuration, a test set of 1000 source locations with signal-to-noise ratio (SNR) in the range from $40 \mathrm{~dB}$ to $100 \mathrm{~dB}$ is generated by varying $\sigma$. Assuming the source signal energy $a$ is known, the SNR is measured at $1 \mathrm{~m}$ from the source by using $10 \log _{10} \frac{a^{2}}{\sigma^{2}}$. For typical applications, the variance of measurement noise amounts to $\sigma^{2}=0.05^{2}(=60 \mathrm{~dB})$ and can be often much higher than $40 \mathrm{~dB}$ for practical vehicle targets [21, 23]. As can be seen in Fig. 3, PDQ performs quite well with respect to the other novel designs in noisy cases.

Table 1 Localization error (LE) of PDQ with $R_{i}=3$ due to variations of the model parameters

\begin{tabular}{llllll}
\hline Decay factor $\alpha$ & 1.8 & 1.9 & 2 & 2.1 & 2.2 \\
LE (PDQ) & 1.5484 & 0.9270 & 0.7038 & 0.9535 & 1.5936 \\
Gain factor $g_{i}$ & 0.8 & 0.9 & 1 & 1.1 & 1.2 \\
LE (PDQ) & 1.2213 & 0.8614 & 0.7017 & 0.7841 & 0.9928 \\
\hline
\end{tabular}

$\mathrm{LE}=\frac{1}{100} \sum_{l=1}^{100} E_{l}\left(\|x-\hat{x}\|^{2}\right)$, where $E_{l}$ is the average localization error for the /th five-sensor configuration and is expressed in meter

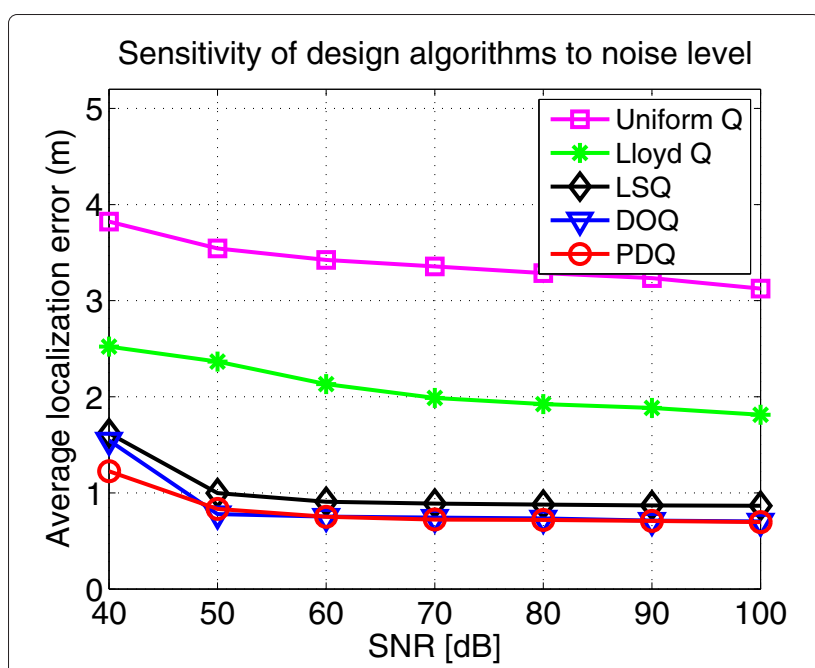

Fig. 3 Sensitivity to noise level. The average localization error is plotted vs. SNR (dB) with $M=5, R_{i}=3$ and $a=50$

\subsection{Performance analysis in a larger sensor network: comparison with traditional quantizers}

In this section, we evaluate our design algorithm in larger sensor networks by comparing with typical designs. In this experiment, we generate 20 different sensor configurations in a larger sensor field, $20 \times 20 \mathrm{~m}^{2}$ for $M=12,16,20$. For each sensor configuration, our quantizers are designed with a given rate of $R_{i}=3$ and the same dynamic range as in the experiments conducted in a $10 \times 10 \mathrm{~m}^{2}$ sensor field. The localization results are provided in Fig. 4. It can be seen that our design algorithm provides very good performance compared with unform quantizers and Lloyd quantizers.

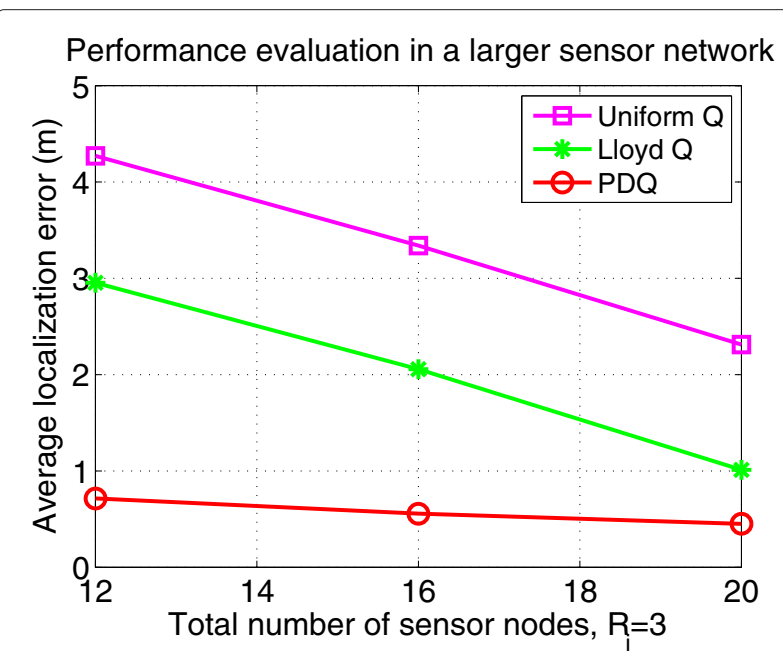

Fig. 4 Performance evaluation in a larger sensor network. The average localization error is plotted vs. the total number of sensor nodes in a $20 \times 20 \mathrm{~m}^{2}$ sensor field with $R_{i}=3$ 
It should be mentioned that better performance can be generally achieved with a larger number of sensors while the sensor density remains unchanged. In our experiments, the sensor density for $M=20$ in $20 \times 20 \mathrm{~m}^{2}$ is given by $\frac{20}{20 \times 20}=0.05$ which is equal to that for the case of $M=5$ in $10 \times 10 \mathrm{~m}^{2}$. This performance gain can be explained by taking into account the coverage of the sensing range of nodes which would become more efficient as the sensor field gets larger. In other words, the sensor nodes located around edges show their poor coverage of the sensing range, leading to performance degradation, and there are a relatively smaller number of sensor nodes near the edge in a larger sensor field as compared to a smaller field with the same sensor density.

\section{Conclusions}

In this paper, we have proposed an iterative quantizer design algorithm that seeks to minimize the probabilistic distance between the posterior distribution and its quantized one. The benefits of our algorithm are illustrated by the analysis that the independent encoding minimizing the global probabilistic distance can be implemented at each node and the global minimum is always guaranteed due to the convexity of the probabilistic distance in our quantizers. In addition, to avoid a computational burden at nodes for encoding process, we have suggested a low-complexity encoding technique which showed a reasonable performance. We demonstrated through extensive experiments that our proposed algorithm achieved a significant performance gain over typical designs and provided a strong competitiveness in comparison with the previous novel designs in terms of performance assessment. In the future, we will continue to develop creative perspectives on the quantization techniques that maximize application objectives for distributed systems.

\section{Acknowledgements}

This research was supported by Basic Science Research Program through the National Research Foundation of Korea (NRF) funded by the Ministry of Education (NRF-2014R1A1A2055997).

\section{Competing interests}

The authors declare that they have no competing interests.

Received: 7 December 2015 Accepted: 22 August 2016

Published online: 02 September 2016

\section{References}

1. SS Pradhan, K Ramchandran, Distributed source coding using syndromes (DISCUS): design and construction. IEEE Trans. Inf. Theory. 49, 626-643 (2003)

2. N Wernersson, J Karlsson, M Skoglund, Distributed quantization over noisy channels. IEEE Trans. Commun. 57, 1693-1700 (2009)

3. A Saxena, J Nayak, K Rose, Robust distributed source coder design by deterministic annealing. IEEE Trans. Signal Process. 58, 859-868 (2010)

4. W Lam, AR Reibman, Design of quantizers for decentralized estimation systems. IEEE Trans. Commun. 41(11), 1602-05 (1993)

5. JA Gubner, Distributed estimation and quantization. IEEE Trans. Inf. Theory. 39(4), 1456-1459 (1993)
6. Z-Q Luo, Universal decentralized estimation in a bandwidth constrained sensor network. IEEE Trans. Inf. Theory. 51(6), 2210-2219 (2005)

7. A Ribeiro, GB Giannakis, Bandwidth-constrained distributed estimation for wireless sensor networks — part ii:unknown probability density function. IEEE Trans. Signal Process. 54(7), 2784-2796 (2006)

8. RM Gray, in IEEE International Conference on Acoustic, Speech, and Signal Processing (ICASSP). Quantization in task-driven sensing and distributed processing (IEEE, Toulouse, 2006)

9. M Longo, TD Lookabaugh, RM Gray, Quantization for decentalized hypothesis testing under communication constraints. IEEE Trans. Inf. Theory. 36(2), 241-255 (1990)

10. HV Poor, Fine quantization in signal detection and estimation. IEEE Trans. Inf. Theory. 34(5), 960-972 (1988)

11. A Hegde, D Erdogmus, T Lehn-Schioler, YN rao, JC Principe, in IEEE International Joint Conference on Neural Networks. Vector-quantization by density matching in the minimum Kullback-Leibler divergence sense (IEEE, 2004)

12. P Venkitasubramaniam, L Tong, A Swami, Quantization for maximum are in distributed estimation. IEEE Trans. Signal Process. 55(7), 3596-3605 (2007)

13. YH Kim, Functional quantizer design for source localization in sensor networks. EURASIP J. Adv. Signal Process. 2013(1), 10 (2013)

14. YH Kim, A Ortega, Quantizer design for energy-based source localization in sensor networks. IEEE Trans. Signal Process. 59(11), 5577-5588 (2011)

15. YH Kim, Weighted distance-based quantization for distributed estimation. J. Inf. Commun. Convergence Eng. 12(4), 215-220 (2014)

16. YH Kim, Maximum likelihood (ML)-based quantizer design for distributed estimation. J. Inf. Commun. Convergence Eng. 13(3), 152-158 (2015)

17. YH Kim, A Ortega, Distributed encoding algorithms for source localization in sensor networks. EURASIP J. Adv. Signal Process. 2010, 13 (2010)

18. YH Kim, Quantizer design optimized for distributed estimation. IEICE Trans. Inf. Systems. E97-D(6), 1639-1643 (2014)

19. YH Kim, Encoding of quantisation partitions optimised for distributed estimation. Electron. Lett. 52(8), 611-613 (2016)

20. TM Cover, JA Thomas, Elements of Information Theory. (Wiley-Interscience Publication, New York, 1991)

21. D Li, YH Hu, Energy-based collaborative source localization using acoustic microsensor array. EURASIP J. Appl. Signal Process. 2003, 321-337 (2003)

22. AO Hero, D Blatt, in IEEE International Conference on Acoustic, Speech, and Signal Processing (ICASSP). Sensor network source localization via projection onto convex sets (POCS) (IEEE, Philadelphia, 2005)

23. J Liu, J Reich, F Zhao, Collaborative in-network processing for target tracking. EURASIP J. Appl. Signal Process. 2003, 378-391 (2003)

24. TS Rappaport, Wireless Communications:principles and Practice. (Prentice-Hall Inc., New Jersey, 1996)

25. YH Kim, A Ortega, in IEEE International Conference on Acoustic, Speech, and Signal Processing (ICASSP). Maximun a posteriori (MAP)-based algorithm for distributed source localization using quantized acoustic sensor readings (IEEE, Toulouse, 2006)

26. YH Kim, A Ortega, in IEEE International Conference on Acoustic, Speech, and Signal Processing (ICASSP). Quantizer design for source localization in sensor networks (IEEE, Philadelphia, 2005) 